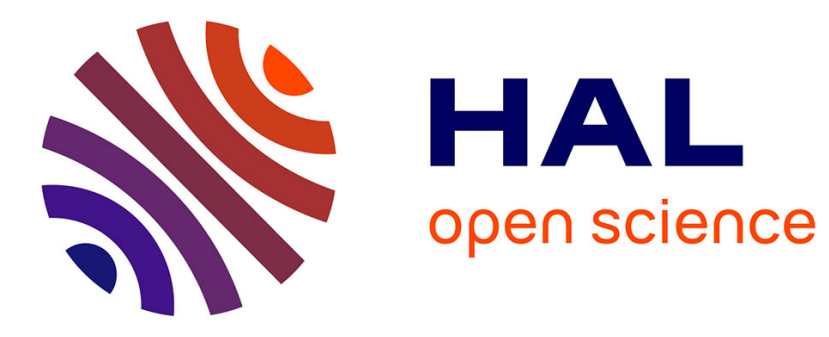

\title{
Personality and Neuropsychological Profiles in Friedreich Ataxia
}

Sabrina Sayah, Jean-Yves Rotgé, Hélène Francisque, Marcela Gargiulo, Virginie Czernecki, Damian Justo, Khadija Lahlou-Laforet, Valérie Hahn, Massimo Pandolfo, Antoine Pelissolo, et al.

\section{To cite this version:}

Sabrina Sayah, Jean-Yves Rotgé, Hélène Francisque, Marcela Gargiulo, Virginie Czernecki, et al.. Personality and Neuropsychological Profiles in Friedreich Ataxia. The Cerebellum, 2018, 17 (2), pp.204-212. 10.1007/s12311-017-0890-5 . hal-01826592

\section{HAL Id: hal-01826592 https: / hal.sorbonne-universite.fr/hal-01826592}

Submitted on 29 Jun 2018

HAL is a multi-disciplinary open access archive for the deposit and dissemination of scientific research documents, whether they are published or not. The documents may come from teaching and research institutions in France or abroad, or from public or private research centers.
L'archive ouverte pluridisciplinaire HAL, est destinée au dépôt et à la diffusion de documents scientifiques de niveau recherche, publiés ou non, émanant des établissements d'enseignement et de recherche français ou étrangers, des laboratoires publics ou privés. 


\title{
Personality and Neuropsychological Profiles in Friedreich Ataxia
}

\author{
Sabrina Sayah ${ }^{1,2} \cdot$ Jean-Yves Rotgé ${ }^{2,3} \cdot$ Hélène Francisque ${ }^{4}$ Marcela Gargiulo ${ }^{1,5,6}$. \\ Virginie Czernecki ${ }^{7}$ • Damian Justo ${ }^{8}$ - Khadija Lahlou-Laforet ${ }^{9}$ - Valérie Hahn ${ }^{8}$. \\ Massimo Pandolfo $^{10}$ - Antoine Pelissolo ${ }^{11}$ - Philippe Fossati ${ }^{2,3}$ - Alexandra Durr ${ }^{1,2,12}$ (iD
}

\begin{abstract}
Friedreich ataxia, an autosomal recessive mitochondrial disease, is the most frequent inherited ataxia. Many studies have attempted to identify cognitive and affective changes associated with the disease, but conflicting results have been obtained, depending on the tests used and because many of the samples studied were very small. We investigated personality and neuropsychological characteristics in a cohort of 47 patients with genetically confirmed disease. The neuropsychological battery assessed multiple cognition domains: processing speed, attention, working memory, executive functions, verbal memory, vocabulary, visual reasoning, emotional recognition, and social cognition. Personality was assessed with the Temperament and Character Inventory, and depressive symptoms were assessed
\end{abstract}

Sabrina Sayah and Jean-Yves Rotgé are joint first authors.

Alexandra Durr

alexandra.durr@upmc.fr

1 AP-HP, Genetic Department, Pitié-Salpêtrière University Hospital, Paris, France

2 ICM, Institut du Cerveau et de la Moelle Epinière, INSERM U1127, CNRS UMR7225, Sorbonne Universités - UPMC Université Paris VI UMR S1127, Paris, France

3 AP-HP, Service de Psychiatrie, Pitié-Salpêtrière University Hospital, Paris, France

4 APHP, Hôpitaux Universitaires Saint Louis Lariboisière Fernand-Widal, Paris, France

5 Institut de Myologie, Pitié-Salpêtrière University Hospital, Paris, France

6 Laboratoire de Psychologie Clinique et Psychopathologie, EA 4056, Université Paris Descartes, Sorbonne Paris Cité, Institut de Psychologie, Paris, France with the Beck Depression Inventory. We found deficits of sustained attention, processing speed, semantic capacities, and verbal fluency only partly attributable to motor deficit or depressed mood. Visual reasoning, memory, and learning were preserved. Emotional processes and social cognition were unimpaired. We also detected a change in automatic processes, such as reading. Personality traits were characterized by high persistence and low self-transcendence. The mild cognitive impairment observed may be a developmental rather than degenerative problem, due to early cerebellum dysfunction, with the impairment of cognitive and emotional processing. Disease manifestations at crucial times for personality development may also have an important impact on personality traits.
7 AP-HP, Département des Maladies du Système Nerveux, Pitié-Salpêtrière University Hospital, Paris, France

8 Unité de neurologie de la Mémoire et du Langage, Centre Hospitalier Sainte-Anne, Paris, France

9 Unité de Psychologie et Psychiatrie de Liaison et d'Urgences, Hôpital Européen Georges Pompidou, Service de Psychiatrie Adulte et du Sujet Agé, Hôpitaux Universitaires Paris-Ouest, Paris, France

10 Service de Neurologie, Hôpital Erasme, Université Libre de Bruxelles, Brussels, Belgium

11 AP-HP, Service de Psychiatrie, Hôpitaux Universitaires Henri-Mondor, Créteil, France

12 ICM, Institut du Cerveau et de la Moelle épinière, Groupe Hospitalier Pitié-Salpêtrière, 47-83 Boulevard de l'Hôpital, 75651 Paris Cedex 13, France 
Keywords Friedreich ataxia - Cerebellum .

Neuropsychology $\cdot$ Personality $\cdot$ Emotional recognition .

Social cognition

\section{Introduction}

Friedreich ataxia (FRDA) is an autosomal recessive disease and the most common inherited form of ataxia. Clinical presentation includes cerebellar and sensory ataxia, associated with pyramidal signs (extensor plantar response and spasticity), an absence of tendon reflexes in the legs, deep sensory loss, and foot deformity. Mean onset of symptoms is around puberty, even if late-onset cases exist [1]. Cardiomyopathy is typically found and is the most common cause of death [2]. Other clinical features, reminiscent of those seen in other mitochondrial diseases, include optic atrophy, deafness, and diabetes. FRDA results from the abnormal expansion of a GAA (guanine, adenine, adenine) triplet repeat in a non-coding region of both alleles of the frataxin $(F X N)$ gene, resulting in the production of only small amounts of frataxin, a small mitochondrial protein $[3,4]$. In less than $2 \%$ of cases, the GAA expansion in one allele is associated with a point mutation [5] or an exonic deletion in the other allele [6]. Age at onset depends partly on expansion size, and onset may occur late, at or beyond the age of about 40 years, in some individuals with short expansions ( $<500$ GAA repeats) [7, 8]. Neuronal loss begins in the dorsal root ganglia, with $\mathrm{T}$ cell degeneration explaining the observed areflexia. Dentate nucleus lesions cause atrophy of the superior cerebellar peduncle, but the Purkinje cell axons are usually preserved [9]. Patients suffer a loss of coordination and gait disorders due to spinal cord degeneration, cerebellar atrophy, or both. FRDA therefore differs from other inherited cerebellar ataxias in which the lesions are found principally in the cerebellum, pons, and cortex.

In addition to its role in motor control, the cerebellum also contributes to non-motor functions. Schmahmann described a cerebellar cognitive affective syndrome (CCAS) consisting of cognitive and affective deficits due to cerebellar disease [10, 11]. CCAS, or Schmahmann syndrome, is characterized by multimodal disturbances, including executive and visuospatial deficits, linguistic impairment, and personality changes [12]. The cerebellum has been shown to play a key role in social cognition, the cognitive processes required to understand, generate, and regulate social behavior [13]. Hoche et al. showed that patients with cerebellar disorders find it difficult to attribute emotions to faces [14]. The recognition of emotions is essential for appropriate social functioning and is a key element of social cognition. Van Overwalle et al. conducted a meta-analysis on social cognition and the cerebellum [15]. They found robust clusters of activation in the cerebellum that were recruited during these social-cognitive processes. A meta-analytic connectivity analysis suggested a key role for the cerebellum in social cognition [16]. Van Overwalle et al. showed that the pattern of activation differed between understanding actions (reading body language) and mentalizing ("mind-reading"), two different aspects of social cognition. Thus, lesions in the cerebellum may cause difficulties with social cognition.

Subtle cognitive and psychiatric abnormalities have been described in FRDA patients [17-21]. A first study of 13 patients showed a higher than normal frequency of personality traits such as increased irritability, poor control over impulses, poor self-presentation, and cognitive dysfunctions characterized by impaired learning processes and verbal fluency. It also showed that mean IQ was lower in FRDA patients than that in controls, this difference being observed for both verbal and performance IQ scores. GAA expansion size was not correlated with neuropsychological performance [17].

Other studies highlighted impairments in verbal fluency [18-20], particularly for tasks requiring major executive processing [21]. Some authors have also reported an impairment of conceptual thinking in FRDA patients [18]. These findings suggest that these patients present prefrontal function deficits. Verbal fluency and working memory deficits have recently been confirmed in a cohort of 22 patients, together with an impairment of social cognition [22].

Long-term follow-up of FRDA patients at the French national reference center for rare diseases defined a recognizable clinical pattern, with increased irritability and poor control of impulses, with consequences for family and social life. We therefore investigated personality and neuropsychological characteristics in a large cohort of patients with genetically confirmed FRDA, through a battery of personality and neuropsychological tests.

\section{Materials and Methods}

\section{Participants}

Between 2012 and 2014, 50 patients with genetically confirmed FRDA were enrolled in the EFACTS (European Friedreich's Ataxia Consortium for Translational Studies, ongoing prospective and longitudinal study, NCT02069509) consortium study. Informed consent was obtained from all individual participants included in the study. Two patients refused to participate and one could not be evaluated due to marked impairment in oral and written communication.

\section{Genetic and Clinical Assessments}

We recorded the number of GAA repeats in each allele (the second allele is the longer allele). Age at onset was assessed retrospectively, according to information provided by patients and their families concerning the first symptoms. Disease 
duration, in years, was calculated as the interval between age at onset and age at examination. Clinical examinations included the determination of a functional disability score ranging from 1 (no functional handicap but signs at examination) to 7 (confined to bed), and SARA (Scale for the Assessment and Rating of Ataxia) score ranging from 0 to 40 .

\section{Personality Assessments}

The Temperament and Character Inventory (TCI) was used to assess the personality dimensions of participants [23, 24]. This 226-item questionnaire deals with seven dimensions of personality traits: four temperament dimensions and three character dimensions. The temperament dimensions considered are novelty seeking (score range $0-40$ ), harm avoidance (score range $0-36$ ), reward dependence (score range $0-24$ ), and persistence (score range $0-8$ ). Novelty seeking targets the tendency of the patient to become excited when presented with novel stimuli. Harm avoidance is the inhibition or cessation of behavior in the face of adverse stimuli (such as pessimistic worry, passive dependent behavior). Reward dependence is a tendency to respond by sentimentality, social attachment, or dependence to signals associated with rewards. Persistence is the tendency to persevere in a particular pattern of behavior despite frustration or fatigue. The character dimensions investigated are self-directedness (score range 0-44), cooperativeness (score range $0-42$ ), and self-transcendence (score range $0-33$ ). Self-directedness is to the ability to control, regulate, and adapt behavior in accordance with personal goals and values. Cooperativeness is the degree of maturity and social tolerability. Self-transcendence relates to spiritual ideas.

\section{Neuropsychological Assessments}

Our neuropsychological battery comprised eight different tests in their French-validated versions:

1. A visual reasoning test was used to measure global cognitive efficiency. The test used was Raven's Progressive Matrices (PM38, SPM), which has been identified as the best instrument for measuring the "g" factor strongly correlated with general mental ability. PM38 is a 60 -item non-timed test, in which each item presents a pattern problem to resolve [25].

2. A vocabulary test was used to assess semantic capacities. The test used was the Mill-Hill part B test $[25,26]$. Participants were asked to select the synonym of the target word from a list of six words. They were presented with 44 target words of increasing difficulty during the test. This multiple choice test was considered to be the most appropriate as it requires a smaller contribution of executive function than part A. Vocabulary is known to increase with age and education level, whereas reasoning ability is considered to be independent of schooling.

3. Verbal learning and memory were assessed with the Hopkins Verbal Learning Test (HVLT) [27]. This test consists of a list of 12 words from three semantic categories (4 words per category). Participants undergo three learning trials each followed by free recall. After the third recall and an interval of 20-25 min, a last recall is performed, followed by a yes-/no-delayed recognition trial.

4. Attentional skills were assessed with the Paced Auditory Serial Addition Test [28, 29], which evaluates sustained attention, short-term memory, working memory, and inhibitory control. Single-digit numbers were presented to the participant at 4-s intervals. The participant had to give the sum of the last two digits before being presented with the next digit. This test had a total duration of $5 \mathrm{~min}$. We evaluated effects on sustained attention, by separating the test into three equal parts and counting the incorrect responses for each part.

5. Focused attention was assessed with the Golden version of the Stroop test, including word reading, color naming, and interference conditions (non-matching words and colors) $[30,31]$. The performance of the Stroop test also requires inhibitory control, and this test can therefore be considered to assess executive functions too. The number of correct responses in $45 \mathrm{~s}$ was recorded.

6. We assessed mental flexibility, by asking participants to give as many words as possible beginning with the letter $P$ (P-fluency test) in a 2-min period. The total number of correct words was recorded.

7. The mini Social Cognitive and Emotional Assessment (SEA) was used to evaluate social cognition and emotional recognition. Social cognition abilities, including theory of mind, in particular, were evaluated with the short version of the Faux-Pas-Recognition test [32-34]. Ten stories were proposed and the participant was asked to explain, for each, whether or not it contained a faux-pas.

8. Emotional recognition was assessed on the basis of the identification of 35 Ekman facial expressions: fear, anger, happiness, surprise, disgust, sadness, neutral [35].

Finally, we checked that neuropsychological performances were not driven by possible depressive symptoms, by assessing the intensity of such symptoms with the Beck Depression Inventory (BDI-II), a 13-item self-administered questionnaire [36].

\section{Data Analyses}

For demographic characteristics and BDI, categories were compared in $\chi^{2}$ tests and means were compared in $t$ tests. The Kolmogorov-Smirnov test was used to evaluate the normality of dependent variables in the whole sample. Three 
variables did not follow a normal distribution: age at onset $(d=0.25, p<0.01$, Lilliefors $p<0.01)$, disability score ( $d=0.29, p<0.01$, Lilliefors $p<0.01)$, and HVLT discrimination $(d=0.48, p<0.01$, Lilliefors $p<0.01)$. One-sample $t$ tests were conducted to compare the mean value for the patient with the reference value. For the personality questionnaire, mean TCI scores for the French population $(n=602)$ were used as reference values. We limited the risk of false-positive results due to the performance of multiple independent statistical tests, by applying Bonferroni correction systematically, to adjust the $p$ values $(p=0.05 / 7=0.007)$. For neuropsychological characteristics, individual scores were compared with French population norms for age and education level. The results were converted into standard scores ( $Z$ scores; i.e., number of standard deviations above or below the mean) as follows: $\{[$ individual score]-[population mean]/[standard deviation of the population]\}. Standard scores took into account gender, age, and educational level. $Z$ scores were then compared with the reference value of 0 . Bonferroni correction was also applied to adjust the $p$ values for multiple analyses $(p=0.05 / 13=0.003)$.

We performed stepwise multiple regression analyses, to explain the possible effects of clinical characteristics on personality and neuropsychological profile. Regression analyses were therefore conducted only when measured values significantly differed from reference values. The following clinical variables were entered into the model as independent variables: age, sex, level of education, age at onset, disease duration, disability score, SARA score, GAA repeats in the shorter allele only (as the smaller allele determines the amount of residual protein and, thus, severity), BDI score. Dependent variables were defined on the basis of the results of onesample $t$ tests with Bonferroni correction. The best multiple regression model was selected by a stepwise approach. The alpha risk threshold was set at 0.05 . Finally, we analyzed the correlation between neuropsychological assessment and personality scores.

\section{Results}

\section{Participants}

In total, 47 patients (29 women and 18 men) gave informed consent for participation in the study. The demographic and clinical characteristics of the participants are presented in Table 1 and Fig. 1 for age at onset distribution. Education level is the number of years spent in education; in France, the Baccalauréat, corresponding to 12 years of education, is the equivalent of the American high-school diploma. Disease manifestations first appeared at or before the age of 16 years in $62 \%$ of patients (29/47). Psychotropic medication was noted in one third of the patients, including six with SSRI, three
Table 1 Demographics and clinical characteristics of FRDA patients $(n=47)$

\begin{tabular}{lrrc}
\hline & Mean & SD & Range \\
\hline Demographics & & & \\
Age (years) & 37.9 & 13.4 & $18-76$ \\
Level of education (years of schooling) & 13.3 & 2.2 & $8-17$ \\
Age at onset (years) & 20.4 & 12.9 & $3-65$ \\
Clinical measurements & & & \\
Disease duration (years) & 16.5 & 8.2 & $4-36$ \\
Disability score (1-7) & 3.7 & 1.5 & $0-5$ \\
SARA score (0-40) & 22.9 & 9.4 & $5-38$ \\
GAA 1 repeats in shorter allele & 472.5 & 253.5 & $90-900$ \\
GAA 2 repeats in longer allele & 890.3 & 210.6 & $250-1250$ \\
\hline
\end{tabular}

$S D$ standard deviation, FRDA Friedreich's ataxia, SARA Scale for the Assessment and Rating of Ataxia

with anxiolytics, three took both. One took lithium and another one neuroleptics in conjunction with SSRI. This is comparable to the psychotropic drug use in the French population (32\% in the report of the French National Agency for Drug Security, ANSM).

\section{Personality Assessments}

FRDA patients had higher scores for persistence temperament $(t=5.38 ; p<0.005)$, but lower scores for the selftranscendence character $(t=-3.13, p<0.005)$ than those of the French general population. No difference between FRDA patients and the general population was observed for other temperament and character dimensions (see $p$ values in Table 2). Self-transcendence and persistence were not

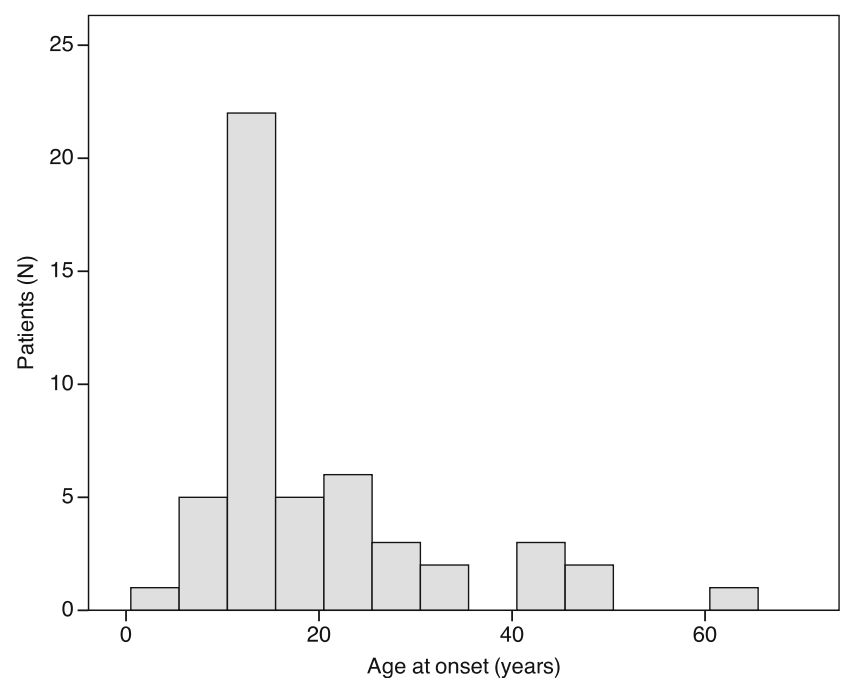

Fig. 1 Age at onset distribution of patients with Friedreich's Ataxia $(n=50)$. The mean age at onset was $20.4 \pm 13.7$ years 
Table 2 Personality evaluation and neuropsychological test performances of FRDA patients

\begin{tabular}{|c|c|c|c|c|c|}
\hline & $n$ & Mean & SD & $t$ & $p$ \\
\hline \multicolumn{6}{|l|}{ Psychopathological evaluation } \\
\hline BDI-II & 33 & 9.8 & 8.3 & - & - \\
\hline \multicolumn{6}{|l|}{ Personality evaluation (TCI) } \\
\hline Novelty seeking $(0-40)$ & 42 & 18.3 & 5.8 & 2.10 & 0.04 \\
\hline Harm avoidance $(0-36)$ & 42 & 18.6 & 7.5 & 2.14 & 0.04 \\
\hline Reward dependence (0-24) & 42 & 15.1 & 3.7 & 1.61 & 0.11 \\
\hline Persistence $(0-8)$ & 42 & 5.4 & 1.7 & 5.38 & $<0.005^{*}$ \\
\hline Self-directedness $(0-44)$ & 42 & 32.8 & 5.2 & 1.00 & 0.32 \\
\hline Cooperativeness $(0-42)$ & 42 & 32.8 & 5.2 & 1.39 & 0.17 \\
\hline Self-transcendence $(0-33)$ & 42 & 10.8 & 5.9 & -3.13 & $<0.005^{*}$ \\
\hline \multicolumn{6}{|c|}{ Neuropsychological test performance } \\
\hline \multicolumn{6}{|l|}{$Z$ scores } \\
\hline Mill-Hill B & 47 & -1.7 & 1.6 & -7.53 & $<0.001 *$ \\
\hline Raven's & 41 & -0.3 & 1.3 & -1.44 & 0.16 \\
\hline HVLT total & 47 & 0.3 & 1.0 & 1.84 & 0.07 \\
\hline PASAT $1 / 3$ & 40 & -0.5 & 1.4 & -2.32 & 0.03 \\
\hline PASAT $2 / 3$ & 40 & -0.7 & 1.7 & -2.64 & 0.01 \\
\hline PASAT $3 / 3$ & 40 & -1.3 & 2.0 & -4.07 & $<0.001 *$ \\
\hline P-fluency & 46 & -0.6 & 1.4 & -2.88 & 0.006 \\
\hline Emotional recognition & 41 & -0.1 & 1.0 & -0.72 & 0.47 \\
\hline Faux-pas & 44 & 0.2 & 1.1 & 1.26 & 0.22 \\
\hline Mini SEA & 41 & 0.1 & 1.2 & 0.59 & 0.56 \\
\hline \multicolumn{6}{|l|}{$T$ scores } \\
\hline Stroop color & 46 & 38.7 & 8.5 & -8.85 & $<0.001 *$ \\
\hline Stroop word & 46 & 36.8 & 8.9 & -9.53 & $<0.001 *$ \\
\hline Stroop interference & 46 & 40.1 & 9.9 & 1.15 & 0.26 \\
\hline
\end{tabular}

$S D$ standard deviation, FRDA Friedreich's ataxia, BDI Beck Depression Inventory, TCI Temperament and Character Inventory, HVLT Hopkins Verbal Learning Test, PASAT Paced Auditory Serial Addition Test, SEA Social Cognition and Emotional Assessment

*Significant after Bonferroni correction

correlated with neuropsychological assessment results (selftranscendence: $F=1.56, R^{2}=0.10, p=0.23$; persistence: $\left.F=2.00, R^{2}=0.18, p=0.14\right)$.

\section{Neuropsychological Assessments}

FRDA patients performed less well than the French general population in the Mill-Hill B test: they had a significantly lower vocabulary level $(t=-7.53, p<0.001)$. Attention was found to be impaired: six of the patients failed the training trials for the Paced Auditory Serial Addition Test (PASAT) and therefore did not perform the full test. The other participants made more errors in the last third of the PASAT $(t=-4.07 ; p<0.001)$ than in the preceding PASAT trials.

Stroop test scores were significantly lower in patients than those in the general population for color $(t=-8.85$; $p<0.001)$ and word reading $(t=-9.53, p<0.001)$, but there was no interference effect $(t=1.15, p=0.26)$. We took the cerebellar dysarthria likely to interfere with Stroop test performances into account by assessing the correlation between the SARA dysarthria item and the scores for the three parts of the Stroop test. Dysarthria was negatively correlated with the scores for all three parts of the Stroop test: color $(r=-0.34 ; p=0.028)$, word reading $(r=-0.41 ; p=0.007)$, and interference $(r=-0.32 ; p=0.042)$.

All other neuropsychological functions, as assessed with Raven's test, and the HVLT, P-fluency, emotional recognition, and faux-pas tests, were similar in patients and in the general population. The results of the neuropsychological assessments are reported in Table 2.

\section{Multiple Regression Analyses}

Regression analyses were conducted to assess the influence of clinical variables on the observed differences. The low Mill-Hill B $Z$ scores obtained were related to sex, age at onset, education level, and BDI score $\left(F=4.68, R^{2}=0.48, p<0.05\right)$. The beta-values, indicative of the direction and strength of the relationship between each independent variable and Mill-Hill B $Z$ scores, were -0.54 for the proportion of women, 0.38 for age at onset, 0.23 for education level, and 0.18 for BDI score. Thus, a more extensive vocabulary was associated with a later age at onset, a higher education level, and also a higher level of depression. In order to inspect the correlation on possible outliers, the correlation with age at onset is shown in Fig. 2a.

Attention performances were related to age (Fig. 2b), sex, disability score, and disease duration $\left(F=3.14, R^{2}=0.42\right.$, $p<0.05)$. Attention in the third part of the test was poorer only in the younger patients $(b=0.28)$, with greater disability $(b=-0.73)$.

In the Stroop test for color, performances were correlated with disability score, disease duration (Fig. 2c), and the number of GAA repeats in the smaller allele $\left(F=8.17, R^{2}=0.48, p<0.05\right)$. The best performances were obtained for patients with lower levels of disability $(b=-0.22)$, shorter disease duration $(b=-0.41)$, and smaller numbers of GAA repeats in the smaller allele was $(b=-0.31)$. For the Stroop word test, performances were correlated with disability score, disease duration (Fig. 2d), and the numbers of GAA repeats in both alleles $(F=7.55$, $\left.R^{2}=0.56, p<0.05\right)$. The best performances for the Stroop word test were obtained for the patients with the lowest disability scores $(b=-0.19)$, the shortest disease duration $(b=-0.43)$, and the smallest numbers of GAA repeats $(b=-0.32)$. 

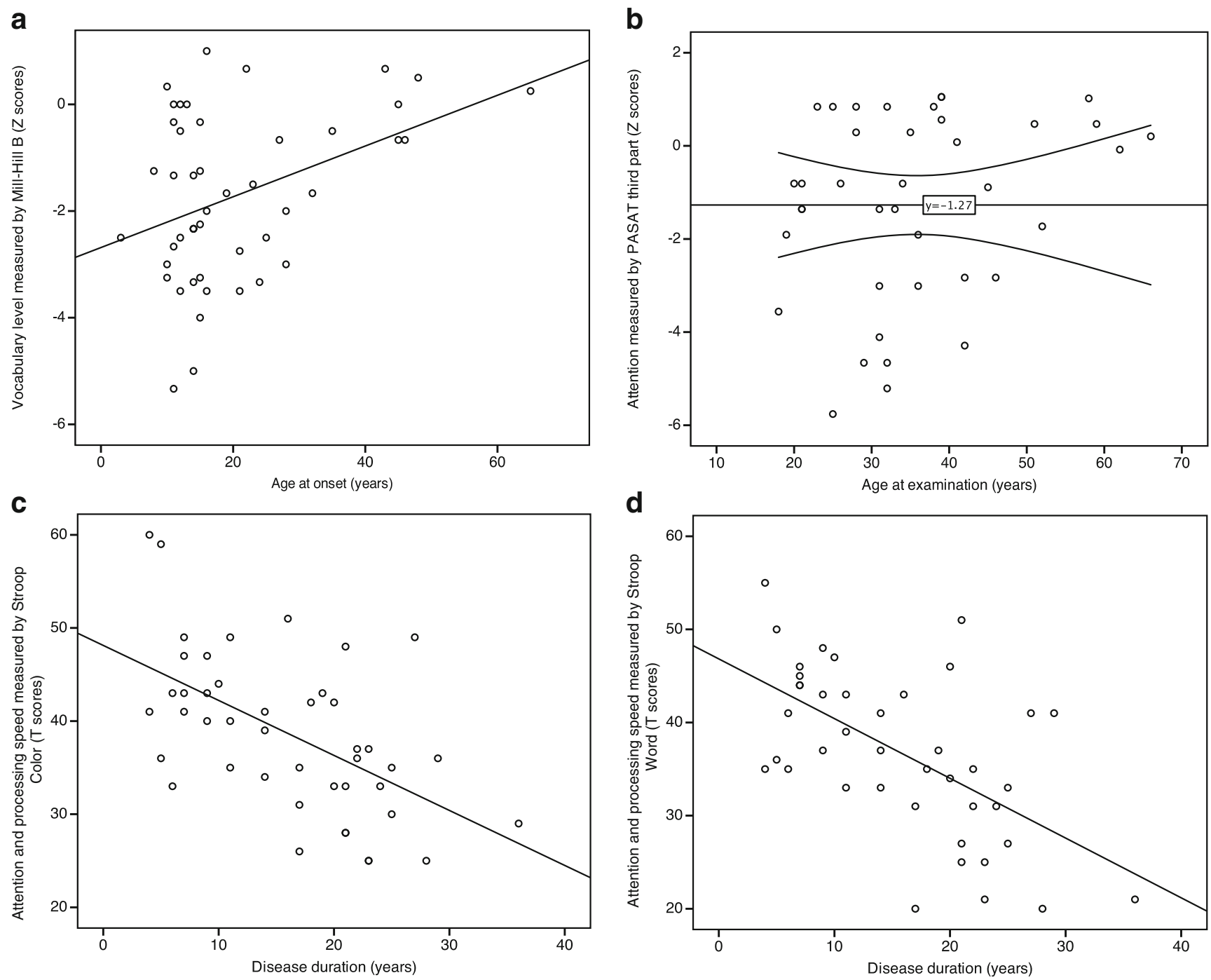

Fig. 2 Correlation scatterplots of four neuropsychological tests (MillHill B, PASAT, Stroop color, and Stroop word) in Friedreich's ataxia. A more extensive vocabulary was associated with a later age at onset $\left(r^{2}=0.156\right)(\mathbf{a})$; attention in the third part of the PASAT was poorer in

\section{Discussion}

This study in a large cohort of FRDA patients assessed personality and neuropsychological profiles and included the first assessment of theory of mind and emotional processes. A comparison of the neuropsychological testing results for 47 FRDA patients with those for the general population revealed deficits in sustained attention, processing speed, semantic capacities, and verbal capacities. Visual reasoning, memory, and learning were preserved. Emotional processes and social cognition were also unimpaired.

Deficits in sustained attention, assessed with the PASAT, were linked to disability score, indicating a direct link to disease severity. Previous studies have given conflicting results concerning volitional sustained attention: one study [18] suggested that sustained attention was preserved, whereas others

the younger patients $\left(r^{2}=0.069\right)(\mathbf{b})$; best performances of processing speed were obtained for patients with shorter disease duration, measured by Stroop color $\left(r^{2}=0.309\right)(\mathbf{c})$ or Stroop word $\left(r^{2}=0.347\right)(\mathbf{d})$

have shown it to be impaired in FRDA patients, with this deficit not due to articulatory deficits [17, 37]. Our study goes one step further, by separating the PASAT into three parts. Those patients able to perform the whole test succeeded in the first two parts of the PASAT, suggesting that these patients have an efficient working memory and a normal capacity to maintain a high level of cognitive effort. Nevertheless, we show here that sustained attention is impaired in FRDA patients, as their time-dependent attention skills were poor.

The Stroop test revealed that patients performed significantly less well than the general population in the color and word tests, whereas there was no difference for the interference test. The results of the three parts of the Stroop test were correlated with dysarthria, suggesting that this condition may account for these results. Our findings confirm that FRDA patients have slower information processing, linked to 
cerebellar dysarthria [20]. Despite this link between dysarthria and the three parts of the Stroop test, patients were able to perform the interference test as well as the general population, whereas they performed poorly in the color and word tests. This very specific pattern in FRDA patients suggested that dysarthria cannot entirely explain the poor performances of these patients in the Stroop test. In the general population, individuals take longer to respond in the color test than in the word test, and their response times increase still further when faced with a color name printed in an incongruent color (e.g., the word "blue" printed in red ink) due to a conflict between the naming of the color and word reading. Indeed, in the general population, reading mechanisms are more automatic than color naming. This conflict seems to be reduced in FRDA patients, allowing them to perform normally in the interference test, despite their dysarthria. Previous studies have demonstrated a link between the cerebellum and reading. Vlachos and colleagues reported such a link, particularly in individuals with reading disabilities [38]. Travis et al. reported an association between reading skills and cerebellar white matter pathways, and, more specifically, with the microstructure of the cerebellar peduncles [39]. Based on the results reported here and published findings, we suggest that the ability to activate automatisms, such as reading, is altered in patients with FRDA.

As expected, semantic capacities were linked to education level. However, comparison with the French general population revealed mild impairment in terms of vocabulary. FRDA patients have been shown to present a deficit in the acquisition of verbal information [18]. This deficit may reflect the effects of articulatory deficits on verbal communication, leading to poorer exchanges and vocabulary acquisition. Nevertheless, the lower verbal and IQ levels in FRDA patients suggest a more global deficit [17]. We found a positive link between Mill-Hill score and age at onset. Semantic capacities were lower in patients with earlier onset disease, but were unrelated to disease duration, consistent with a significant contribution of the cerebellum to the development of cognitive abilities.

We found a trend towards lower verbal fluency in FRDA patients. It might be possible to establish a link with information processing speed capacities by considering the time dependence of the test. However, dysarthria is not the only cause of the decrease in processing speed [17, 18, 20, 22, 40,41]. The impairment of phonemic and action fluencies may, therefore, be better explained by the strong executive component of these tasks requiring shifting abilities, because semantic fluency was unimpaired [40, 42].

The neuropsychological profile of the FRDA patients in this study was associated with a specific personality presentation characterized by enhanced persistence and reduced selftranscendence. A limitation of questionnaires is that selfreport may obscure impairments of socioemotional functioning due to lack of self-insight. The fact that the patients score lower on self-transcendence may hint towards this. Typically, socioemotional problems arise in the context of social interactions or daily life activities. Observational scores from, for example, family or friends may be a better way to capture problems in this domain. Nevertheless, our study is the first exploring both, social cognition and emotional recognition, and there was no impairment of theory of mind in FRDA. This allowed us to take into account the low scores of selfrated transcendence. The personality assessment revealed that patients were more likely to persevere in the face of fatigue or frustration than members of the general population. Selftranscendence is a personality trait relating to the experience of spiritual aspects of the self. It is seen as an identification of the self with a unified whole. FRDA-induced uncertainty about the future, disability, and shortened life expectancy may bring out feelings of otherness, making this identification with a unified whole more difficult. Previous studies have shown that self-transcendence is associated with self-wellbeing and adaptation [43, 44]. Some authors have reported that high scores for the self-transcendence trait characterize individuals open to unusual and divergent feelings, thoughts, and behaviors [45]. However, persistence and transcendence were not correlated with clinical characteristics and neuropsychological data. It should be borne in mind that disease manifestations often begin during adolescence. The diagnosis of a chronic disease at this crucial age may influence personality development [46]. However, contrary to our findings, chronic diseases have consistently been shown to be associated with high levels of harm avoidance and low self-directedness and cooperativeness [47-49], suggesting that the personality changes observed here are more specific to FRDA. We can therefore assume that the observed changes are also linked to the FRDA-related lesions of cerebellar pathways. This idea is consistent with the cognitive and affective functions of the cerebellum $[10,50]$. A close relationship between cerebellar volumes and personality traits in a healthy population has been reported, suggesting a role for the cerebellum in personality development [51-53]. However, further studies are required to assess specifically the putative relationship between personality changes and cerebellar lesions in FRDA.

The main limitation of this study was the absence of a control group matched for age, sex, and number of years spent in education. However, this bias was minimized by comparing our results with mean values for a very large French general population for the TCI, and with standardized scores for neuropsychological measures. This strategy involved the use of multiple independent statistical tests, potentially increasing the risk of false-positive results. We therefore systematically applied Bonferroni correction, the most conservative method for controlling for the risk of type I error. However, it should be acknowledged that the lack of impairments was due to type II errors. As an example, the significant lower fluency did not survive correction but we felt that the difference was clinically 
important. In addition, our population had an older mean age at onset than reported (20.4 \pm 13.7 versus $15.5 \pm 10.4$ in a 2 year cohort study [54] that makes generalization of our results more hazardous. In fact, patients with onset after 25 years may have different phenotypic and brain structural features compared to classical FRDA [55].

The cognitive impairment and the specific personality profile in FRDA patients demonstrated here provide additional evidence concerning the role of the cerebellum in cognitive and affective processing and personality development. Compared to other ataxias, such as SCA3, similar cognitive processes are impaired in FRDA, such as attention, processing speed, and executive functions [56]. The neuropsychological dysfunctions in FRDA are clearly milder despite a higher motor disability. Imaging comparison between SCA3 and FRDA showed that volume of the cerebellum is not reduced in FRDA compared to that of SCA3 [57]. Therefore, attribution of FRDA-related cognitive deficits exclusively to CCAS is difficult, even though there were functional MRI abnormalities in the cerebellar cortex in both groups.

\section{Conclusion}

In conclusion, FRDA patients had a specific personality and neuropsychological profile. Our findings highlight the presence of very mild cognitive impairment in a large cohort of patients with a genetically confirmed diagnosis. The neuropsychological profile was characterized by a low vocabulary level, changes in automatisms, such as reading, and deficits in sustained attention, and processing speed. The FRDA-related personality traits, characterized by high persistence and low self-transcendence, may be linked to this particular neuropsychological profile.

Acknowledgments This study is an ancillary substudy of EFACTS (European Friedreich Ataxia Consortium for Translational Studies www.e-facts.eu, NCT02069509) coordinated by M. Pandolfo. We thank the participants for their patience, and Aurelie Guignebert, Martine Frischmann, and Mathieu Anheim for fruitful initial discussions.

\section{Compliance with Ethical Standards}

Conflict of Interest The authors declare that they have no conflict of interest.

Ethical Approval All procedures performed in studies involving human participants were carried out in accordance with the ethical standards of the institutional and/or national research committee and the 1964 Helsinki declaration and its later amendments or comparable ethical standards.

\section{References}

1. Parkinson MH, Boesch S, Nachbauer W, Mariotti C, Giunti P. Clinical features of Friedreich's ataxia: classical and atypical phenotypes. J Neurochem. 2013;126(s1):103-17.

2. Pousset F, Legrand L, Monin M-L, Ewenczyk C, Charles P, Komajda M, et al. A 22-year follow-up study of long-term cardiac outcome and predictors of survival in Friedreich ataxia. JAMA Neurol. 2015;72(11):1334-41.

3. Campuzano V, Montermini L, Moltò MD, Pianese L, et al. Friedreich's ataxia: autosomal recessive disease caused by an intronic GAA triplet repeat expansion. Science. 1996;271(5254): 1423.

4. Campuzano V, Montermini L, Lutz Y, Cova L, Hindelang C, Jiralerspong S, et al. Frataxin is reduced in Friedreich ataxia patients and is associated with mitochondrial membranes. Hum Mol Genet. 1997;6(11):1771-80.

5. Cossée M, Dürr A, Schmitt M, Dahl N, Trouillas P, Allinson P, et al. Friedreich's ataxia: point mutations and clinical presentation of compound heterozygotes. Ann Neurol. 1999;45(2):200-6.

6. Anheim M, Mariani L-L, Calvas P, Cheuret E, Zagnoli F, Odent S, et al. Exonic deletions of FXN and early-onset Friedreich ataxia. Arch Neurol. 2012;69(7):912-6.

7. Dürr A, Cossee M, Agid Y, Campuzano V, Mignard C, Penet C, et al. Clinical and genetic abnormalities in patients with Friedreich's ataxia. N Engl J Med. 1996;335(16):1169-75.

8. Lecocq C, Charles P, Azulay J-P, Meissner W, Rai M, N'Guyen K, et al. Delayed-onset Friedreich's ataxia revisited. Mov Disord. 2016;31(1):62-9.

9. Koeppen AH, Mazurkiewicz JE. Friedreich ataxia: neuropathology revised. J Neuropathol Exp Neurol. 2013;72(2):78-90.

10. Schmahmann JD, Sherman JC. The cerebellar cognitive affective syndrome. Brain J Neurol. 1998;121(Pt 4):561-79.

11. Manto M, Mariën P. Schmahmann's syndrome - identification of the third cornerstone of clinical ataxiology. Cerebellum Ataxias. 2015;2:2.

12. Schmahmann JD, Weilburg JB, Sherman JC. The neuropsychiatry of the cerebellum - insights from the clinic. Cerebellum. 2007;6(3): 254-67.

13. Fiske ST, Taylor SE. Social cognition: from brains to culture. 2nd edition. Los Angeles: Sage; 2013.

14. Hoche F, Guell X, Sherman JC, Vangel MG, Schmahmann JD. Cerebellar contribution to social cognition. Cerebellum. 2015; $1-$ 12.

15. Van Overwalle F, Baetens K, Mariën P, Vandekerckhove M. Social cognition and the cerebellum: a meta-analysis of over $350 \mathrm{fMRI}$ studies. NeuroImage. 2014;86:554-72.

16. Van Overwalle F, D'aes T, Mariën P. Social cognition and the cerebellum: a meta-analytic connectivity analysis. Hum Brain Mapp. 2015;36(12):5137-54.

17. Mantovan MC, Martinuzzi A, Squarzanti F, Bolla A, Silvestri I, Liessi G, et al. Exploring mental status in Friedreich's ataxia: a combined neuropsychological, behavioral and neuroimaging study. Eur J Neurol Off J Eur Fed Neurol Soc. 2006;13(8):827-35.

18. Nieto A, Correia R, de Nóbrega E, Montón F, Hess S, Barroso J. Cognition in Friedreich ataxia. Cerebellum. 2012;11(4):834- 44.

19. Nieto A, Correia R, de Nóbrega E, Montón F, Barroso J. Cognition in late-onset Friedreich ataxia. Cerebellum. 2013;12(4):504-12.

20. Wollmann T, Barroso J, Monton F, Nieto A. Neuropsychological test performance of patients with Friedreich's ataxia. J Clin Exp Neuropsychol. 2002;24(5):677-86.

21. de Nóbrega E, Nieto A, Barroso J, Montón F. Differential impairment in semantic, phonemic, and action fluency performance in Friedreich's ataxia: possible evidence of prefrontal dysfunction. J Int Neuropsychol Soc. 2007;13(6):944-52. 
22. Dogan I, Tinnemann E, Romanzetti S, Mirzazade S, Costa AS, Werner CJ, et al. Cognition in Friedreich's ataxia: a behavioral and multimodal imaging study. Ann Clin Transl Neurol. 2016;3(8):572-87.

23. Cloninger CR, Przybeck TR, Svrakic DM. The Temperament and Character Inventory (TCI): a guide to its development and use. Center for Psychobiology of Personality, Washington University St. Louis; 1994.

24. Pélissolo A, Lépine J-P. Normative data and factor structure of the Temperament and Character Inventory (TCI) in the French version. Psychiatry Res. 2000;94(1):67-76.

25. Raven JC, Court JH. Raven's progressive matrices and vocabulary scales. Oxford: Oxford Psychologists Press; 1998.

26. Deltour JJ. Echelle de vocabulaire de Mill Hill de JC Raven. Adapt Fr Normes Eur Mill Hill Stand Progress Matrices Raven PM38 Braine-Château Ed L'application Tech Mod. 1993.

27. Rieu D, Bachoud-Lévi A-C, Laurent A, Jurion E, Dalla BG. Adaptation française du «Hopkins verbal learning test». Rev Neurol (Paris). 2006;162(6):721-8.

28. Gronwall D, Sampson H. The psychological effects of concussion. New York: Oxford University Press; 1974.

29. Naegele B, Mazza S. Test d'attention auditive soutenue PASAT modifié. De Boeck Supérieur: Louvain-La-Neuve; 2013.

30. Golden CJ. Stroop color and word test. Chicago: Stoelting Co.; 1978.

31. Stroop JR. Studies of interference in serial verbal reactions. J Exp Psychol. 1935;18(6):643.

32. Gregory C, Lough S, Stone V, Erzinclioglu S, Martin L, BaronCohen $\mathrm{S}$, et al. Theory of mind in patients with frontal variant frontotemporal dementia and Alzheimer's disease: theoretical and practical implications. Brain. 2002;125(4):752-64.

33. Stone VE, Baron-Cohen S, Knight RT. Frontal lobe contributions to theory of mind. J Cogn Neurosci. 1998;10(5):640-56.

34. Funkiewiez A, Bertoux M, de Souza LC, Lévy R, Dubois B. The SEA (Social cognition and Emotional Assessment): a clinical neuropsychological tool for early diagnosis of frontal variant of frontotemporal lobar degeneration. Neuropsychology. 2012;26(1): 81.

35. Ekman P, Friesen WV. Pictures of facial affect. Palo Alto: Consulting Psychologists Press; 1975.

36. Beck AT, Steer RA, Brown GK. Inventaire de dépression de Beck. 2ème édition ed. Paris: les Éditions du centre de psychologie appliquée; 1998

37. Klopper F, Delatycki MB, Corben LA, Bradshaw JL, Rance G, Georgiou-Karistianis N. The test of everyday attention reveals significant sustained volitional attention and working memory deficits in Friedreich ataxia. J Int Neuropsychol Soc. 2011;17(01):196-200.

38. Vlachos F, Papathanasiou I, Andreou G. Cerebellum and reading. Folia Phoniatr Logop. 2007;59(4):177-83.

39. Travis KE, Leitner Y, Feldman HM, Ben-Shachar M. Cerebellar white matter pathways are associated with reading skills in children and adolescents. Hum Brain Mapp. 2015;36(4):1536-53.

40. De NÓbrega É, Nieto A, Barroso J, MontÓn F. Differential impairment in semantic, phonemic, and action fluency performance in Friedreich's ataxia: possible evidence of prefrontal dysfunction. J Int Neuropsychol Soc. 2007;13(06):944-52.
41. Nachbauer W, Bodner T, Boesch S, Karner E, Eigentler A, Neier L, et al. Friedreich ataxia: executive control is related to disease onset and GAA repeat length. Cerebellum. 2014;13(1):9-16.

42. White M, Lalonde R, Botez-Marquard T. Neuropsychologic and neuropsychiatric characteristics of patients with Friedreich's ataxia. Acta Neurol Scand. 2000;102(4):222-6.

43. Cloninger CR, Zohar AH. Personality and the perception of health and happiness. J Affect Disord. 2011;128(1):24-32.

44. Cloninger CR, Svrakic DM, Przybeck TR. A psychobiological model of temperament and character. Arch Gen Psychiatry. 1993;50(12):975-90.

45. Kaasinen V, Maguire RP, Kurki T, Brück A, Rinne JO. Mapping brain structure and personality in late adulthood. NeuroImage. 2005;24(2):315-22.

46. Jokela M, Hakulinen C, Singh-Manoux A, Kivimäki M. Personality change associated with chronic diseases: pooled analysis of four prospective cohort studies. Psychol Med. 2014;44(12): 2629-40.

47. Boz C, Gazioglu S, Altunayoglu V, Hocaoglu C. Effect of serotonergic antidepressant therapy on temperament and character scales in patients with chronic tension-type headache. Psychiatry Clin Neurosci. 2007;61(5):534-42.

48. Conrad R, Schilling G, Bausch C, Nadstawek J, Wartenberg HC, Wegener I, et al. Temperament and character personality profiles and personality disorders in chronic pain patients. Pain. 2007;133(1-3):197-209.

49. Kahraman H, Orhan FO, Sucakli MH, Ozer A, Koksal N, Sen B. Temperament and character profiles of male COPD patients. J Thorac Dis. 2013;5(4):406-13.

50. Dolan RJ. A cognitive affective role for the cerebellum. Brain. 1998;121(4):545-6.

51. Picerni E, Petrosini L, Piras F, Laricchiuta D, Cutuli D, Chiapponi C, et al. New evidence for the cerebellar involvement in personality traits. Front Behav Neurosci. 2013;7:133.

52. Laricchiuta D, Petrosini L, Piras F, Macci E, Cutuli D, Chiapponi C, et al. Linking novelty seeking and harm avoidance personality traits to cerebellar volumes. Hum Brain Mapp. 2014;35(1):285-96.

53. Petrosini L, Cutuli D, Picerni E, Laricchiuta D. Cerebellum and personality traits. Cerebellum. 2015;14(1):43-6.

54. Reetz K, Dogan I, Hilgers R-D, Giunti P, Mariotti C, Durr A, et al. Progression characteristics of the European Friedreich's Ataxia Consortium for Translational Studies (EFACTS): a 2 year cohort study. Lancet Neurol. 2016;15(13):1346-54.

55. Rezende TJR, Martinez ARM, Faber I, Girotto K, Pedroso JL, Barsottini OG, et al. Structural signature of classical versus lateonset friedreich's ataxia by multimodality brain MRI. Hum Brain Mapp. 2017;38(8):4157-68.

56. Braga-Neto P, Pedroso JL, Alessi H, Dutra LA, Felício AC, Minett $\mathrm{T}$, et al. Cerebellar cognitive affective syndrome in Machado Joseph disease: core clinical features. Cerebellum. 2012;11(2):549-56.

57. Stefanescu MR, Dohnalek M, Maderwald S, Thürling M, Minnerop $\mathrm{M}$, Beck A, et al. Structural and functional MRI abnormalities of cerebellar cortex and nuclei in SCA3, SCA6 and Friedreich's ataxia. Brain. 2015;138(5):1182-97. 